Conclusions Antifungal spending is disproportionately high considering the low number of fungal isolates, and entails a high use of empirical and prophylactic treatment.

Haematology is, by far, the main department responsible for the use of antifungal treatment. Consumption of voriconazole and liposomal amphotericin B are increasing, meanwhile caspofungin is decreasing in recent years.

No conflict of interest.

\section{DGI-037 FINGOLIMOD IN RELAPSING REMITTING MULTIPLE SCLEROSIS: A CASE REPORT}

doi:10.1136/ejhpharm-2013-000276.303

'M Merchante, ' 'A Izquierdo, 'S Martinez, 'AP Zorzano, 'L Sanchez-Rubio, 'A Serrano, 'MF Hurtado, 'MA Alfaro, 2 Cañamares. 'Hospital San Pedro, Hospital Pharmacy, Logroño, Spain; ${ }^{2}$ Hospital Laprincesa, Hospital Pharmacy, Madrid, Spain

Background Fingolimod has recently been authorised in our country (April 2011). It is the first orally administered diseasemodifying drug that has been approved for highly active relapsing remitting multiple sclerosis. So far, only one patient has been treated with it in our hospital, so we have limited experience in its use.

Purpose The case report relates to relapsing remitting multiple sclerosis (RRMS) patient with high disease activity under treatment with Fingolimod. We aim to describe the evolution of this patient during the treatment period.

Materials and Methods It was an observational, six-month prospective study.

The patient, a 32-year-old female, was diagnosed with RRMS in February 2004 after an episode of sensory deficits.

Results At first, she was treated with interferon b-1a, which was stopped in February 2006 and switched to mitoxantrone IV. The patient continued to have several relapses during the treatment with this immunosuppressant; one of these relapses required plasma exchange therapy. Her Expanded Disability Status Scale (EDSS) worsened to 6 points. Assuming a lack of efficacy, the patient started treatment with natalizumab in April 2007. During four years of treatment with natalizumab she showed remarkable clinical improvement and did not experience any new relapses. Her EDSS improved to 2.5. After this time and due to the high risk of developing progressive multifocal leukoencephalopathy (PML), she switched to fingolimod (December 2011).

Ten days after initiation, she developed a severe relapse that required hospital admission, high dose IV steroids and 3 cycles of plasma exchange therapy. Doctors concluded this relapse was in fact a rebound effect due to stopping natalizumab.

In February 2012 she restarted fingolimod; one month later she developed a new relapse, treated with high dose steroids.

In April and May 2012 she had two more relapses, with severe EDSS worsening and again managed with high dose steroids.

In May 2012, it was decided to stop treatment with fingolimod, and despite the risk of PML (JC virus +), natalizumab was restarted. Conclusions During six months of fingolimod treatment, the patient's condition further deteriorated (four relapses in six months), her EDSS worsened and showed a high disease activity. We conclude that the treatment was not effective in this patient.

No conflict of interest.

\section{DGl-038 GEMTUZUMAB OZOGAMICIN AS SALVAGE TREATMENT IN CHILDREN WITH ACUTE MYELOID LEUKAEMIA RELAPSE: A RETROSPECTIVE STUDY}

doi:10.1136/ejhpharm-2013-000276.304

${ }^{1} \mathrm{~A}$ Giroud, ' $\mathrm{K}$ Morand, 'G Benoit, ${ }^{2} \mathrm{G}$ Leverger. 'Armand Trousseau Hospital, Pharmacy, Paris, France; ${ }^{2}$ Armand Trousseau Hospital, Pediatric oncology/haematology, Paris, France
Background Gemtuzumab ozogamicin (GO) is a humanised antiCD33 monoclonal antibody conjugated with calicheamicin. Several studies show its safety and efficacy in refractory/relapsed acute myeloid leukaemia (AML). Nevertheless in July 2010 it was withdrawn from the US market after a study failed to confirm the clinical benefits of GO.

Purpose Following this controversy, we conducted a retrospective study to evaluate its efficacy and safety in children with refractory/ relapsed AML.

Materials and Methods The study focused on the 19 children treated after approval by the French drug safety agency, between October 2006 and June 2012.

Results The median age at initial diagnosis was 6.7 years (0.5-15.3). Three patients were refractory to first-line treatment, one patient was in refractory first relapse, three were in first relapse after stem cell transplantation (SCT), three in second relapse after SCT, one in third relapse after SCT, seven were in first relapse and one in second relapse. Patients received: one dose of $3 \mathrm{mg} / \mathrm{m}^{2}$ with cytarabine (day 1 to 7 ); or $9 \mathrm{mg} / \mathrm{m}^{2}$ fractionated dose (on days 1 , 4,7 ) in monotherapy or associated with cytarabine (day 1 to 7 ); or $4.5 \mathrm{mg} / \mathrm{m}^{2}$ on day 6 associated with fludarabine and daunorubicin liposomal. Nine complete remissions were obtained (48\%) in 32 days, leading to further curative treatment. The one year overall survival was $26 \%$ (5 patients). For the others complete remission was maintained for 6-9 months before relapse or death. Grade 3-4 haematological adverse events were identified in all children including severe thrombocytopenia requiring transfusion. Sepsis $(n=2)$, fever $(n=3)$, vomiting $(n=6)$ were documented. One case of sinusoidal obstruction syndrome was reported.

Conclusions Children with refractory/relapsed AML have a dismal outcome and there is a lack of effective treatments. In our cohort GO led to nearly $50 \%$ of CRs and even if the long term survival is still unsatisfactory it should remain available in this indication.

No conflict of interest.

\section{DGI-039 GUIDE TO THE PREPARATION AND ADMINISTRATION OF INJECTABLE CYTOTOXIC DRUGS}

doi:10.1136/ejhpharm-2013-000276.305

${ }^{1} \mathrm{M}$ Morgado, ${ }^{2} \mathrm{~A}$ Pinto, ${ }^{2 \mathrm{R}}$ Oliveira, 'S Morgado. 'Hospital Centre of Cova da Beira, Pharmaceutical Services, Covilhã, Portugal; ${ }^{2}$ University of Beira Interior, Health Sciences Faculty, Covilhã, Portugal

Background The preparation of injectable cytotoxics is a key activity of many hospital pharmaceutical services. Due to the increasing availability of cytotoxic medicines, either branded or generic, the time spent by hospital pharmacists in search of information about reconstitution and/or dilution, storage and stability of these drugs has increased. In order to effectively respond to this need for information, it would be useful to have a database that holds all that information for all cytotoxic medicines currently available in Portugal.

Purpose To prepare a guide to the preparation and administration of all parenterally administered cytotoxics available in Portugal, which provides information on the reconstitution and/or dilution, storage and stability, routes of administration, infusion rate, as well as other relevant observations.

Materials and Methods Review of the summary of product characteristics (SPC) of all injectable cytotoxic drugs currently available in Portugal; consultation with the pharmaceutical manufacturers and analysis of the responses received.

Results A total of 153 injectable cytotoxic medicines were investigated ( 88 branded and 65 generic), comprising a total of 40 active substances. Of this total, 145 have marketing authorization in Portugal and 8 are used under special-use authorization. Significant variability in the information available about the reconstitution, 